\section{Article}

ULLAH, R. ${ }^{1}$

ASLAM, Z. ${ }^{1 *}$

KHALIQ, A. ${ }^{1}$

ZAHIR, Z.A. ${ }^{2}$

\title{
SUNFLOWER RESIDUE INCORPORATION SUPPRESSES WeEd, ENhaNCEs SoIl Properties ANd SEed YIELd OF SPRING-Planted MUNG BeAN
}

\author{
A incorporação de resíduos de girassol suprime plantas daninhas, melhora as \\ propriedades do solo e o rendimento de sementes do girassol plantado na \\ primavera
}

\begin{abstract}
Continuous and excessive use of herbicides to control various weed biotypes is posing severe threats to soil and human health. Nevertheless, the strategy of using allelopathic crop residues and water extracts for weed control seems to be a sustainable and friendly option to reduce dependence on synthetic herbicides. In addition to controlling weeds, residues of allelopathic crops positively affected soil health. A two-year field study was carried out to examine the impact of sunflower residues on soil properties, weed dynamics and productivity of mung bean. The study was comprised of five treatments viz., control, sunflower water extracts at 10 and $20 \mathrm{~L} \mathrm{ha}^{-1}$, and sunflower residues incorporation at 4 and 6 ton ha- $\mathrm{h}^{-1}$. Results indicated that application of sunflower water extracts reduced weed density by $5-26 \%$ and weed dry weight by $9-31 \%$, while sunflower residue incorporation caused $44-57 \%$ reduction in weed density and $58-70 \%$ reduction in weed dry weight compared with the control. At mung bean harvest, total nitrogen and organic matter in soil were increased by $86 \%$ and $74 \%$, respectively, with the incorporation of sunflower residues at 6 ton ha ${ }^{-1}$ as compared with the control. In case of soil enzymes, alkaline phosphatase and dehydrogenase were recorded at $186 \mathrm{mg} \mathrm{NP} \mathrm{g}^{-1} \mathrm{soil} \mathrm{h}^{-1}$ and $38 \mathrm{mg} \mathrm{TPF} \mathrm{g}^{-1}$ soil h$^{-1}$, respectively, when sunflower residue was incorporated at 6 ton ha- ${ }^{-1}$. In conclusion, sunflower residue incorporation at 6 ton ha- ${ }^{-1}$ improved soil health, suppressed weeds and resulted in better seed yield (36\%) and profitability $\left(339 \$\right.$ ha $\left.^{-1}\right)$ of spring-planted mung bean.
\end{abstract}

Keywords: allelopathy, soil fertility, soil enzymes, crop residues.

* Corresponding author:
<zauaf@hotmail.com>

Received: May 4, 2017

Approved: July 15, 2017

\section{Planta Daninha 2018; v36:e018176393}

Copyright: This is an open-access article distributed under the terms of the Creative Commons Attribution License, which permits unrestricted use, distribution, and reproduction in any medium, provided that the original author and source are credited.

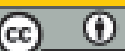

RESUMO - O uso contínuo e excessivo de herbicidas para controle de vários biótipos de plantas daninhas tem causado graves ameaças ao solo e à saúde humana. No entanto, a estratégia de utilizar resíduos culturais e extratos aquosos de efeito alelopático para o controle de plantas daninhas parece ser uma opção sustentável e benéfica para reduzir a dependência do uso de herbicidas sintéticos. Além de controlar plantas daninhas, os resíduos culturais de efeito alelopático exerceram efeito positivo na saúde do solo. Um estudo de campo com duração de dois anos foi realizado para examinar o impacto dos resíduos de girassol nas propriedades do solo, na dinâmica das plantas daninhas e na produtividade do feijão-mungo. $O$ estudo foi composto por cinco tratamentos: controle, extratos aquosos de girassol a 10 e $20 \mathrm{~L} \mathrm{ha}^{-1}$ e incorporação de resíduos de girassol a 4 e

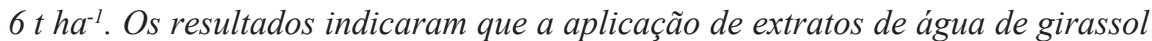
reduziu a densidade de plantas daninhas em 5-26\% e o peso seco delas em 9-31\%, enquanto a incorporação de resíduos de girassol causou redução de 44-57\% na

1 Department of Agronomy, University of Agriculture, Faisalabad-38040, Pakistan; ${ }^{2}$ Institute of Soil and Environmental Sciences, University of Agriculture, Faisalabad-38040, Pakistan. 
densidade de plantas daninhas e redução de 58-70\% no peso seco dessas plantas, em comparação com o controle. Na colheita de feijão-mungo, o nitrogênio e a matéria orgânica totais no solo aumentaram $86 \%$ e 74\%, respectivamente, com a incorporação de residuos de girassol a 6 t ha $\mathrm{h}^{-1}$, em relação ao controle. No caso de enzimas do solo, a fosfatase alcalina e a desidrogenase foram registradas a

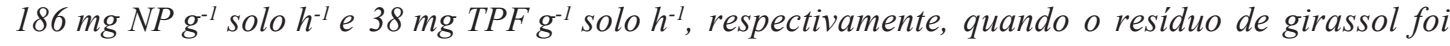
incorporado a 6 ton $h a^{-1}$. Em conclusão, a incorporação de resíduos de girassol a 6 t ha $a^{-1}$ causou a melhoria da saúde do solo e a supressão das plantas daninhas, e obteve melhor rendimento de sementes $(36 \%)$ e rentabilidade (\$339 $\left.\mathrm{ha}^{-1}\right)$ de feijão-mungo plantado na primavera.

Palavras-chave: alelopatia, fertilidade do solo, enzimas do solo, resíduos culturais.

\section{INTRODUCTION}

The world's human population crossed the 7.3 billion mark in 2015 and is estimated by the United Nations to reach 8.4 billion by 2030 and 9.6 billion by 2050 . Food production will have to be increased by $40 \%$ to $70 \%$ in order to feed the growing numbers by 2050 (World Resources Institute, 2014). Amazingly, food production was increased by 146\% from 1961 to 2000, while available agricultural land was increased by only $8 \%$ (Bai et al., 2008). This was attained through intensive use of inputs such as nutrients and agrochemicals. However, this intensification of agricultural lands has increased soil degradation, erosion, depletion of nutrients and micro flora as well as greenhouse gases emissions (Johnson et al., 2007; Hussain et al., 2015).

Mung bean (Vigna radiata L.) belongs to the Fabaceae (Leguminosae) family and is cultivated both in the spring and summer seasons. In Pakistan, mung bean is an important pulse crop and covers an area of 146.3 thousand hectares with an average yield of 9.8 ton ha ${ }^{-1}$, which is lesser than world's potential yield (Government of Pakistan, 2016). Weed infestation is one of the major bottlenecks for higher mung bean productivity (Aktar et al., 2015). Weeds are problematic because of their faster growth and competition for use of natural resources (such as light, air, space, moisture and nutrients) and, consequently, interference with uniform growth (Hussain et al., 2015; Fahad et al., 2015). Horse purslane (Trianthema portulacastrum L.) and Purple nutsedge (Cyperus rotundus L.) are major weeds in mung bean fields of Pakistan (Ashiq and Cheema, 2005). Long season weed-crop competition reduces green pod yield by $45.60 \%$ (Pandey and Mishra, 2003).

Currently, as a result of the intensification of crops and quick weed control, the trend of using chemical herbicides is increasing day by day (Khaliq et al., 2013). In underdeveloped countries, the chances of misuse of agrochemicals are comparatively high as compared to developed ones because there is less awareness about safety measures for agrochemicals; low literacy rate and lack of skills to handle and apply farm chemicals as per recommendations (Tariq et al., 2007). This is creating many problems about their use. Intensive use of herbicides for weed management is a severe ecological and environmental risk to the planet and its inhabitants. The effects of herbicides on soil and ground water quality, shifts in weed population, weed resistance, and associated health problems have diverted the focus of research toward determining and establishing alternative tools for weed management. To find out alternative strategies for weed management which can reduce the dependence upon traditional practices and synthetic herbicides, time is required (Farooq et al., 2011). Therefore, all approaches that improve crop growth and prevent weed germination and growth must be combined for the best results (Fahad et al., 2015). Allelopathic crops have strong potential for the development of cultivars that are highly weed-suppressive (Weston and Duke, 2003). Incorporation of allelopathic crop residues suppressed weeds through physical hindrance or by posing chemical (allelopathy) effects and improved soil health (Khaliq et al., 2015). Crop residues are good sources of nutrients and the primary source of organic material added to soil. They increase the nutrient and water holding capacity of soils by reducing water loss through evapotranspiration (Krishna et al., 2004). Moreover, incorporation of crop residues can also nourish rhizosphere biota as well as reduce farm inputs and greenhouse gases emissions (Malhi and Lemke, 2007; Sharma et al., 2011). Along with weed suppression, residue incorporation also provides various additional benefits, 
e.g., slow release of nutrients, lighter soil color (which reflects light), and cooler soil temperature (Bajgai et al., 2015).

Weed suppression by the use of sunflower water extract, sunflower residues or inclusion of sunflower in rotation has been well documented in the past (Hozayn et al., 2011). However, little is known about the effect of such uses of allelopathic interventions on soil properties (Kong et al., 2008; Wu et al., 2009). Therefore, the present study was carried out to ascertain the effects of sunflower water extracts and sunflower residues on weeds, soil nutrient dynamics, soil enzyme activities, microbial populations, and seed yield of mung bean.

\section{MATERIALS AND METHODS}

\section{Site description}

The experiment was conducted on the Student Research Farm, Department of Agronomy, University of Agriculture Faisalabad, Pakistan. According to the USDA (US Department of Agriculture) classification, it is an arid fine-silty, hyperan thermic Ustalfic, mixed and Haplargid soil, but in FAO's (Food and Agriculture Organization) classification system, it was classified as a Haplic Yermosol. Figure 1 shows the data recorded on weather parameters during the whole period of crop growth.

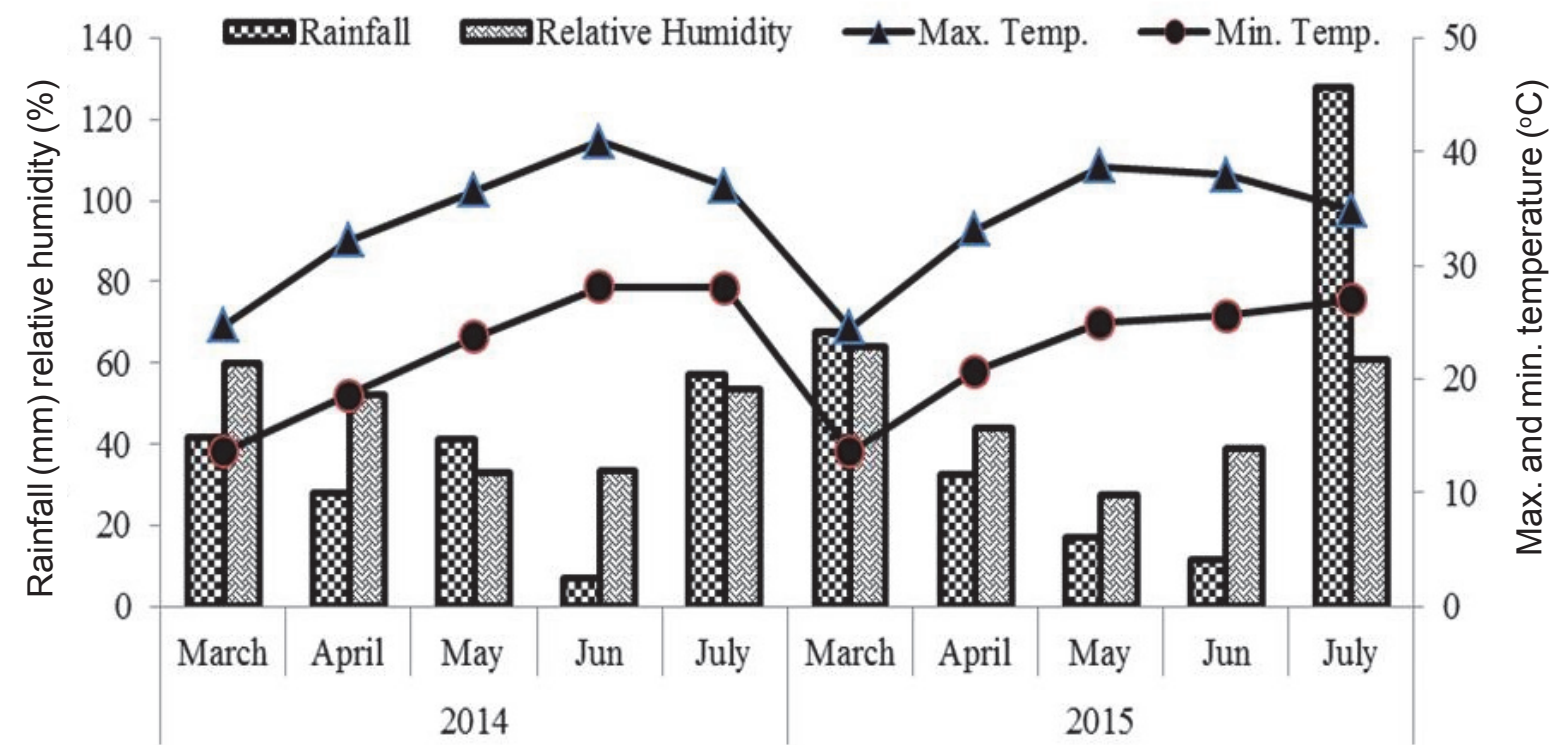

Figure 1 - Monthly meteorological data during the course of the present study (2014 and 2015).

\section{Treatments and experimental design}

The treatments were comprised of a control (plots with no crop residues or extract application), sunflower water extract at $10 \mathrm{~L} \mathrm{ha}^{-1}$, sunflower water extract at $20 \mathrm{~L} \mathrm{ha}^{-1}$, sunflower residue incorporation at 4 ton $\mathrm{ha}^{-1}$, and sunflower residue incorporation at 6 ton ha ${ }^{-1}$. The experiment was laid out in a randomized complete block design (RCBD) with 3 replications. Plot size was maintained at $3.0 \mathrm{~m} \times 5.0 \mathrm{~m}$.

Sunflower plant residues were collected from the Student Research Farm. The plants were harvested at maturity, dried under shady conditions and cut into $2-3 \mathrm{~cm}$ pieces with the help of an electric fodder cutter. These pieces of crop residues were incorporated prior to sowing as per treatments ( 4 and 6 ton $\mathrm{ha}^{-1}$ ). For preparation of water extracts, the pieces of sunflower residues were put into distilled water at a ratio of $1: 10 \mathrm{w} / \mathrm{v}$ for 24 hours. The filtrate was used as fresh. Sunflower water extracts at 10 and $20 \mathrm{~L} \mathrm{ha}^{-1}$ were sprayed 15 days after sowing (DAS) using a T-jet nozzle. Spray volume was $300 \mathrm{~L} \mathrm{ha}^{-1}$. 


\section{Crop husbandry}

Conventional tillage, including ploughing the field twice with a tractor-mounted plough followed by planking, was used to attain mandatory tilth. The seed of mung bean cultivar "NM-92" was used. Sowing was performed on March 15th and March 20 th in 2014 and 2015, respectively. A recommended rate of mung bean seed $\left(25 \mathrm{~kg} \mathrm{ha}^{-1}\right)$ was used to maintain plant population $\left(350,000\right.$ plants ha $\left.{ }^{-1}\right)$ in $30 \mathrm{~cm}$-apart rows. Nitrogen $(\mathrm{N})$, phosphorus $(\mathrm{P})$ and potash $(\mathrm{K})$ fertilizers were applied at $23 \mathrm{~kg} \mathrm{~N}, 58 \mathrm{~kg} \mathrm{P}_{2} \mathrm{O}_{5}$ and $63 \mathrm{~kg} \mathrm{~K}_{2} \mathrm{O}$ ha-1 in the form of Urea and Di-ammonium Phosphate (DAP) and Sulphate of Potash (SOP), respectively. Recommended full doses of P, K and one third of $\mathrm{N}$ in the form of DAP, SOP and Urea, respectively, were drilled at sowing time. Two thirds of the $\mathrm{N}$ rate was applied in two equal splits, i.e. $1 / 3$ at first irrigation and the remaining $1 / 3^{\text {rd }}$ as top dressing at second irrigation. All other agronomic practices except those under study were kept the same for all experimental units.

\section{Rhizosphere soil sampling}

Twenty days after sowing and at the time of harvesting, samples were collected from ten plants. Plants were uprooted safely, and rhizosphere soil was obtained from the root surface by lifting the root from the soil and shaking the whole root system manually. After passing through a $2 \mathrm{~mm}$ sieve, the soil was mixed. Freshly harvested soil was used to determine microbial population and soil enzymatic activity but dried soil was used to analyze soil properties (Ruiyu et al., 2007). Table 1 shows the initial properties of the original experimental site that was tested.

Table 1 - Response of soil properties, nutrient dynamics, soil enzyme activities and microbial populations of the experimental soil before sowing (2014 and 2015)

\begin{tabular}{|l|r|r|}
\hline Soil properties, nutrient dynamics, soil enzyme activities and microbial populations & 2014 & 2015 \\
\hline Soil bulk density $\left(\mathrm{g} \mathrm{cm}^{-3}\right)$ & 1.48 & 1.45 \\
\hline Total soil porosity $(\%)$ & 43.1 & 44.3 \\
\hline Soil pH & 7.85 & 7.79 \\
\hline Electrical conductivity $\left(\mathrm{dS} \mathrm{m}^{-1}\right)$ & 1.11 & 1.19 \\
\hline Total soil organic matter $(\%)$ & 0.53 & 0.61 \\
\hline Available phosphorus $\left(\mathrm{mg} \mathrm{kg}^{-1}\right)$ & 6.74 & 6.95 \\
\hline Available potassium $\left(\mathrm{mg} \mathrm{kg}^{-1}\right)$ & 123 & 131 \\
\hline Total soil nitrogen $\left(\mathrm{g} \mathrm{kg}^{-1}\right)$ & 0.24 & 0.29 \\
\hline Bacteria $(\mathrm{cfu} / \mathrm{g} \mathrm{x} \mathrm{10})$ & 35 & 45 \\
\hline Fungi $\left(\mathrm{cfu} / \mathrm{g} \times 10^{4}\right)$ & 5 & 8 \\
\hline Alkaline phosphatase activity $\left(\mu \mathrm{g} \mathrm{N} \mathrm{g} \mathrm{g}^{-1} \mathrm{soil} \mathrm{h}^{-1}\right)$ & 135 & 143 \\
\hline Dehydrogenase activity $\left(\mu \mathrm{g} \mathrm{TPF} \mathrm{g} \mathrm{soil} \mathrm{h}^{-1}\right)$ & 21 & 25 \\
\hline
\end{tabular}

\section{Data recorded}

\section{Soil properties and nutrient dynamics}

Soil physical properties viz., soil porosity and bulk density, were determined by the method described by Vomocil (1965) and Blake and Hartge (1986), respectively. Soil pH and EC were determined as described by Ryan et al. (2001). For determination of soil pH and EC, 1:2 ratio (soil/water; w/v) of suspension was used. Soil $\mathrm{pH}$ value was recorded by a $\mathrm{pH}$ meter (model Kent Eil 7015, US Salinity Laboratory Staff, 1954). The EC value was determined with a digital Jenway conductivity meter (model 4510, US Salinity Laboratory Staff, 1954). Soil nitrogen (N), available phosphorus (P), available potassium (K), and soil organic matter (SOM) were determined through the method described by Bremner and Mulvaney (1982), Olsen and Sommers (1982), Helmke and Sparks (1996), and Walkley and Black (1934), respectively. 


\section{Microbial populations and soil enzymatic activities}

Microbial populations were determined by spiral plating serial dilutions of each soil sample on agar plates. The total number of culturable bacteria was determined on $\mathrm{R}_{2} \mathrm{~A}$ (half-strength) agar plates (Janssen et al., 2002; Wu et al., 2004; Aslam et al., 2008) and all culturable fungi were plated on rose bengal potato dextrose agar (Martin, 1950). Colony counts were carried out 48 hours after culturing. Dehydrogenase enzymatic activity was determined by the method described by Min et al. (2001). It was expressed as $\mu \mathrm{g}$ TPF g $\mathrm{g}^{-1} 12 \mathrm{~h}^{-1}$. Alkaline phosphatase activity was measured spectrophotometrically as suggested by Tabatabai and Bremner (1969). It was expressed as $\mu \mathrm{g}$ p-nitrophenol $\mathrm{g}^{-1} \mathrm{~h}^{-1}$.

\section{Weed dynamics}

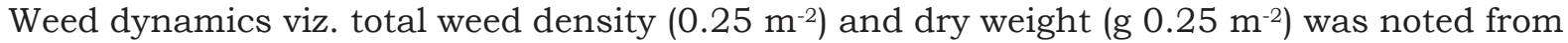
each plot at 30 DAS by randomly selecting two quadrates $(50 \mathrm{~cm} \times 50 \mathrm{~cm})$. After counting weed density, the aboveground parts of weeds were harvested for measurement of dry weight. Firstly, the weed samples were sun-dried for $48 \mathrm{hrs}$, and were further placed in an electric oven for 72 hours at $70{ }^{\circ} \mathrm{C}$ temperature. After attaining constant weight, dry weight was recorded by electric balance.

\section{Yield components}

Yield components (number of pods per plant, number of seeds per pod and 1000-seed weight) were recorded as described by Rab et al. (2016). The crop was harvested at maturity and manual threshing was performed to separate the seeds from the straw. Seed yield of each experimental unit was noted and transformed into $\mathrm{kg} \mathrm{ha}^{-1}$.

\section{Data analysis}

Statistical analysis of data was performed by using Statistix 8.1 (Analytical computer software, Statistix 8.1; Tallahassee, F.L., USA, 1985-2003) and RCBD (randomized complete block design) with factorial arrangement by considering year as a factor. To compare the treatments means, the least significance difference (LSD) test at 5\% probability was applied (Steel et al., 1996).

\section{RESULTS AND DISCUSSION}

\section{Soil properties and nutrients dynamics}

Soil properties and nutrients status are the best indication of soil health. This study showed that soil porosity, bulk density, $\mathrm{pH}, \mathrm{EC}$ (electrical conductivity), SOM (soil organic matter), $\mathrm{N}$ (nitrogen), available K (potassium) and $\mathrm{P}$ (phosphorus) were significantly different among various allelopathic weed management strategies (Table 2). The year effect was also statistically significant for soil porosity, bulk density, $\mathrm{pH}, \mathrm{EC}$, SOM, N, available $\mathrm{K}$ and $\mathrm{P}$ (Table 2). The interaction (allelopathic weed management strategies $\times$ year) was statistically significant for soil porosity, bulk density, SOM, $\mathrm{N}$ and available P. However, for soil $\mathrm{pH}$, EC and available K, interaction was non-significant (Table 2).

The lowest bulk density $\left(1.26 \mathrm{~g} \mathrm{~cm}^{-3}\right)$ and the highest soil porosity $(48.49 \%)$ were found in the $2^{\text {nd }}$ year, when sunflower residues at 6 ton ha $^{-1}$ were applied, as compared to the control. The statistically highest soil bulk density $\left(1.48 \mathrm{~g} \mathrm{~cm}^{-3}\right)$ was found in the control, and it was statistically similar to that of the sunflower water extracts at 10 and $20 \mathrm{~L} \mathrm{ha}^{-1}$ (Table 2). In the case of nutrient dynamics, the highest values of SOM (1.32\%), N $\left(0.42 \mathrm{~g} \mathrm{~kg}^{-1}\right)$ and available P $\left(10.18 \mathrm{mg} \mathrm{kg}^{-1}\right)$ were found in the $2^{\text {nd }}$ year, when sunflower residues at 6 ton ha $^{-1}$ were applied as compared to the control $\left(0.69 \%, 0.21 \mathrm{~g} \mathrm{~kg}^{-1}\right.$ and $6.78 \mathrm{mg} \mathrm{kg}^{-1}$, respectively). Among all allelopathic weed management strategies, the statistically highest values of soil EC $\left(1.32 \mathrm{ds} \mathrm{m}^{-1}\right)$ and available $\mathrm{K}$ 
ULLAH, R. et al. Sunflower residue incorporation suppresses weeds, enhances soil properties and seed yield of ...

Table 2 - Influence of sunflower water extract and residues on soil properties and nutrient dynamics in the rhizosphere of mung bean aton harvest

\begin{tabular}{|c|c|c|c|c|c|c|}
\hline \multirow{2}{*}{ Treatment } & 2014 & 2015 & Mean $(\mathrm{T})(4)$ & 2014 & 2015 & Mean $(\mathrm{T})$ \\
\hline & \multicolumn{3}{|c|}{ Soil bulk density $\left(\mathrm{g} \mathrm{cm}^{-3}\right)$} & \multicolumn{3}{|c|}{ Total soil porosity (\%) } \\
\hline Control $^{(1)}$ & $1.49 \mathrm{a}$ & $1.47 \mathrm{a}$ & $1.48 \mathrm{~A}$ & $43.15 \mathrm{c}$ & $44.06 \mathrm{c}$ & $43.61 \mathrm{C}$ \\
\hline SFWE at $10 \mathrm{~L} \mathrm{ha}^{-1(2)}$ & $1.48 \mathrm{a}$ & $1.47 \mathrm{a}$ & $1.48 \mathrm{~A}$ & $43.87 \mathrm{c}$ & $44.43 \mathrm{bc}$ & $44.15 \mathrm{C}$ \\
\hline SFWE at $20 \mathrm{~L} \mathrm{ha}^{-1}$ & $1.48 \mathrm{a}$ & $1.47 \mathrm{a}$ & $1.48 \mathrm{~A}$ & $44.13 \mathrm{c}$ & $44.45 \mathrm{bc}$ & $44.29 \mathrm{C}$ \\
\hline SFR at 4 ton $\mathrm{ha}^{-1(3)}$ & $1.44 \mathrm{ab}$ & $1.32 \mathrm{bc}$ & $1.38 \mathrm{~B}$ & $45.76 \mathrm{~b}$ & $47.72 \mathrm{a}$ & $46.74 \mathrm{~B}$ \\
\hline SFR at 6 ton $\mathrm{ha}^{-1}$ & $1.41 \mathrm{ab}$ & $1.26 \mathrm{c}$ & $1.33 \mathrm{C}$ & $45.96 \mathrm{~b}$ & $48.49 \mathrm{a}$ & $47.22 \mathrm{~A}$ \\
\hline Mean $(Y)^{(5)}$ & $1.46 \mathrm{~A}$ & $1.40 \mathrm{~B}$ & & $44.57 \mathrm{~B}$ & $45.83 \mathrm{~A}$ & \\
\hline \multirow[t]{2}{*}{$\operatorname{LSD}(\mathrm{p} \leq 0.05)$} & \multicolumn{3}{|c|}{$\mathrm{T}=0.04 ; \mathrm{Y}=0.05 ; \mathrm{T} \times \mathrm{Y}=0.11$} & \multicolumn{3}{|c|}{$\mathrm{T}=1.53 ; \mathrm{Y}=0.72 ; \mathrm{T} \times \mathrm{Y}=1.60$} \\
\hline & \multicolumn{3}{|c|}{ Soil pH } & \multicolumn{3}{|c|}{ Soil EC $\left(\mathrm{dS} \mathrm{m}^{-1}\right)$} \\
\hline Control & 7.78 & 7.75 & $7.77 \mathrm{~A}$ & 1.07 & 1.11 & $1.09 \mathrm{C}$ \\
\hline SFWE at $10 \mathrm{~L} \mathrm{ha}^{-1}$ & 7.78 & 7.74 & $7.76 \mathrm{~A}$ & 1.11 & 1.14 & $1.13 \mathrm{BC}$ \\
\hline SFWE at $20 \mathrm{~L} \mathrm{ha}^{-1}$ & 7.77 & 7.73 & $7.75 \mathrm{~A}$ & 1.13 & 1.16 & $1.15 \mathrm{BC}$ \\
\hline SFR at 4 ton $\mathrm{ha}^{-1}$ & 7.47 & 7.44 & $7.46 \mathrm{~B}$ & 1.21 & 1.25 & $1.23 \mathrm{AB}$ \\
\hline SFR at 6 ton $\mathrm{ha}^{-1}$ & 7.41 & 7.21 & $7.31 \mathrm{C}$ & 1.30 & 1.34 & $1.32 \mathrm{~A}$ \\
\hline Mean $(\mathrm{Y})$ & 7.64 & 7.57 & & $1.17 \mathrm{~B}$ & $1.20 \mathrm{~A}$ & \\
\hline \multirow[t]{2}{*}{$\operatorname{LSD}(p \leq 0.05)$} & \multicolumn{3}{|c|}{$\mathrm{T}=0.13$} & \multicolumn{3}{|c|}{$\mathrm{T}=0.12 ; \mathrm{Y}=0.02$} \\
\hline & \multicolumn{3}{|c|}{ Total soil organic matter (\%) } & \multicolumn{3}{|c|}{ Total soil nitrogen $\left(\mathrm{g} \mathrm{kg}^{-1}\right)$} \\
\hline Control & $0.68 \mathrm{~d}$ & $0.69 \mathrm{~d}$ & $0.69 \mathrm{C}$ & $0.21 \mathrm{~d}$ & $0.21 \mathrm{~d}$ & $0.21 \mathrm{C}$ \\
\hline SFWE at $10 \mathrm{~L} \mathrm{ha}^{-1}$ & $0.68 \mathrm{~d}$ & $0.69 \mathrm{~d}$ & $0.69 \mathrm{C}$ & $0.21 \mathrm{~d}$ & $0.21 \mathrm{~d}$ & $0.21 \mathrm{C}$ \\
\hline SFWE at $20 \mathrm{~L} \mathrm{ha}^{-1}$ & $0.69 \mathrm{~d}$ & $0.71 \mathrm{~d}$ & $0.70 \mathrm{C}$ & $0.21 \mathrm{~d}$ & $0.21 \mathrm{~d}$ & $0.21 \mathrm{C}$ \\
\hline SFR at 4 ton ha ${ }^{-1}$ & $0.91 \mathrm{c}$ & $1.19 \mathrm{ab}$ & $1.05 \mathrm{~B}$ & $0.29 \mathrm{c}$ & $0.35 \mathrm{~b}$ & $0.32 \mathrm{~B}$ \\
\hline SFR at 6 ton ha ${ }^{-1}$ & $1.08 \mathrm{~b}$ & $1.32 \mathrm{a}$ & $1.20 \mathrm{~A}$ & $0.35 \mathrm{~b}$ & $0.42 \mathrm{a}$ & $0.39 \mathrm{~A}$ \\
\hline Mean $(\mathrm{Y})$ & $0.81 \mathrm{~B}$ & $0.92 \mathrm{~A}$ & & $0.25 \mathrm{~B}$ & $0.28 \mathrm{~A}$ & \\
\hline \multirow[t]{2}{*}{$\operatorname{LSD}(p \leq 0.05)$} & \multicolumn{3}{|c|}{$\mathrm{T}=0.09 ; \mathrm{Y}=0.06 ; \mathrm{T} \times \mathrm{Y}=0.14$} & \multicolumn{3}{|c|}{$\mathrm{T}=0.02 ; \mathrm{Y}=0.02 ; \mathrm{T} \times \mathrm{Y}=0.04$} \\
\hline & \multicolumn{3}{|c|}{ Available potassium $\left(\mathrm{mg} \mathrm{kg}^{-1}\right)$} & \multicolumn{3}{|c|}{ Available phosphorous $\left(\mathrm{mg} \mathrm{kg}^{-1}\right)$} \\
\hline Control & 122.12 & 123.26 & $122.69 \mathrm{C}$ & $6.75 \mathrm{~d}$ & $6.78 \mathrm{~d}$ & $6.77 \mathrm{C}$ \\
\hline SFWE at $10 \mathrm{~L} \mathrm{ha}^{-1}$ & 122.18 & 123.33 & $122.76 \mathrm{C}$ & $6.78 \mathrm{~d}$ & $6.80 \mathrm{~d}$ & $6.79 \mathrm{C}$ \\
\hline SFWE at $20 \mathrm{~L} \mathrm{ha}^{-1}$ & 122.18 & 123.33 & $122.76 \mathrm{C}$ & $6.79 \mathrm{~d}$ & $6.81 \mathrm{~d}$ & $6.80 \mathrm{C}$ \\
\hline SFR at 4 ton ha ${ }^{-1}$ & 173.85 & 185.00 & $179.43 \mathrm{~B}$ & $7.99 \mathrm{c}$ & $9.15 \mathrm{~b}$ & $8.57 \mathrm{~B}$ \\
\hline SFR at 6 ton ha ${ }^{-1}$ & 190.00 & 201.67 & $195.83 \mathrm{~A}$ & $9.15 \mathrm{~b}$ & $10.18 \mathrm{a}$ & $9.67 \mathrm{~A}$ \\
\hline Mean $(\mathrm{Y})$ & $146.07 \mathrm{~B}$ & $151.32 \mathrm{~A}$ & & $7.46 \mathrm{~B}$ & $7.95 \mathrm{~A}$ & \\
\hline $\operatorname{LSD}(p \leq 0.05)$ & \multicolumn{3}{|c|}{$\mathrm{T}=8.16 ; \mathrm{Y}=5.16$} & \multicolumn{3}{|c|}{$\mathrm{T}=0.42 ; \mathrm{Y}=0.26 ; \mathrm{T} \times \mathrm{Y}=0.59$} \\
\hline
\end{tabular}

Figures of interaction and main effects sharing the same case letter, do not differ significantly $(\mathrm{p} \leq 0.05)$ by the least significant difference test; likewise, the figures of main effects and interaction without lettering, do not differ significantly ( $\mathrm{p} \leq 0.05)$ by the least significant difference test; ${ }^{(1)}$ Control= plots with no crop residues or extract application; ${ }^{(2)} \mathrm{SFWE}=$ sunflower water extract; ${ }^{(3)} \mathrm{SFR}=$ sunflower residues; (4) $\mathrm{T}=$ treatments; ${ }^{(5)} \mathrm{Y}=$ year.

(195.83 $\left.\mathrm{mg} \mathrm{kg}^{-1}\right)$ were found after application of sunflower residues at 6 ton ha-1. The statistically lowest $\left(1.09 \mathrm{ds} \mathrm{m}^{-1}\right.$ and $\left.122.69 \mathrm{mg} \mathrm{kg}^{-1}\right)$ values for all parameters given above were found in the control, and it was statistically similar to that of the sunflower water extracts at 10 and $20 \mathrm{~L} \mathrm{ha}^{-1}$ (Table 2). A linear increase in soil EC and available $\mathrm{K}$ was found over time and these parameters (soil EC and available K) had the highest values during the $2^{\text {nd }}$ year of the experiment (Table 2). In case of soil $\mathrm{pH}$, there was a decreasing trend. The lowest soil $\mathrm{pH}$ (7.31) was found after application of sunflower residues at 6 ton $\mathrm{ha}^{-1}$ and the highest soil $\mathrm{pH}(7.77)$ was found in the control, and it was statistically similar to that of the sunflower water extracts at $10 \mathrm{~L} \mathrm{ha}^{-1}$ and $20 \mathrm{~L} \mathrm{ha}^{-1}$ (Table 2).

\section{Microbial population and soil enzymatic activities}

Microbial abundance and soil enzymes are robust indicators of soil fertility. Microbial populations at 20 days after sowing and at harvesting, soil enzymatic activities (alkaline 
phosphatase and dehydrogenase) differed significantly among various allelopathic weed management strategies (Table 3). The year effect was also significant for all above parameters (Table 3). The interactive effect of allelopathic weed management strategies and year was significant for alkaline phosphatase and dehydrogenase activity but it was non-significant for the population of bacteria and fungi (Table 3).

Among the all allelopathic weed management strategies, the highest bacterial $\left(75 \mathrm{cfu} / \mathrm{g} \times 10^{5}\right.$ and $39 \mathrm{cfu} / \mathrm{g} \times 10^{5}$, respectively) and fungal populations $\left(21 \mathrm{cfu} / \mathrm{g} \times 10^{4}\right.$ and $15 \mathrm{cfu} / \mathrm{g} \times 10^{4}$, respectively) were recorded after application of sunflower residues at 6 ton ha ${ }^{-1}$ at both stages i.e., 20 days after sowing and at harvesting. The lowest populations were found in the control; they were statistically similar with sunflower water extracts at 10 and $20 \mathrm{~L} \mathrm{ha}^{-1}$, Table 3). There was a linear increase in bacterial and fungal populations at 20 days after sowing and at harvesting over time and the highest bacterial and fungal populations were found in the second year but the fungal population at 20 days after sowing was statistically the same in both years (Table 3). In the case of soil enzymes, the interactive effect of allelopathic weed management strategies and year resulted in a significant effect on the activity of both alkaline phosphatase and dehydrogenase enzyme. The highest values of alkaline phosphatase $\left(191.23 \mu \mathrm{g} \mathrm{NP} \mathrm{g}{ }^{-1} \mathrm{soil} \mathrm{h}^{-1}\right)$ and dehydrogenase $\left(41.00 \mu \mathrm{g}\right.$ TPF g ${ }^{-1}$ soil h$\left.^{-1}\right)$ were found after the application of sunflower residues at 6 ton ha ${ }^{1}$ in the $2^{\text {nd }}$ year, followed by the same treatment in the $1^{\text {st }}$ year. The lowest values of alkaline phosphatase (135.15 $\mu \mathrm{g} \mathrm{NP} \mathrm{g}{ }^{-1}$ soil h$\left.^{-1}\right)$ and dehydrogenase $\left(23.00 \mu \mathrm{g}\right.$ TPF g ${ }^{-1}$ soil h$\left.^{-1}\right)$ were recorded in the control (Table 3).

Table 3 - Influence of sunflower water extract and residues on microbial population and soil enzymatic activity in the rhizosphere of mung bean

\begin{tabular}{|c|c|c|c|c|c|c|}
\hline \multirow{2}{*}{ Treatment } & 2014 & 2015 & Mean $(\mathrm{T})^{(4)}$ & 2014 & 2015 & Mean $(\mathrm{T})$ \\
\hline & \multicolumn{3}{|c|}{ Bacteria $\left(\mathrm{cfu} \mathrm{g}^{-1} \times 10^{5}\right) 20$ DAS $^{(6)}$} & \multicolumn{3}{|c|}{ Fungi $\left(\mathrm{cfu} \mathrm{g}^{-1} \times 10^{4}\right) 20$ DAS } \\
\hline Control $^{(1)}$ & 43 & 44 & $44 \mathrm{C}$ & 7 & 8 & $8 \mathrm{C}$ \\
\hline SFWE at $10 \mathrm{~L} \mathrm{ha}^{-1(2)}$ & 43 & 44 & $44 \mathrm{C}$ & 7 & 8 & $8 \mathrm{C}$ \\
\hline SFWE at $20 \mathrm{~L} \mathrm{ha}^{-1}$ & 44 & 45 & $45 \mathrm{C}$ & 8 & 9 & $9 \mathrm{C}$ \\
\hline SFR at 4 ton $\mathrm{ha}^{-1(3)}$ & 60 & 70 & $65 \mathrm{~B}$ & 13 & 18 & $15 \mathrm{~B}$ \\
\hline SFR at 6 ton $\mathrm{ha}^{-1}$ & 70 & 79 & $75 \mathrm{~A}$ & 19 & 23 & $21 \mathrm{~A}$ \\
\hline Mean $(Y)^{(5)}$ & $52 \mathrm{~B}$ & $56 \mathrm{~A}$ & & $11 \mathrm{~B}$ & $13 \mathrm{~A}$ & \\
\hline \multirow[t]{2}{*}{$\operatorname{LSD}(\mathrm{p} \leq 0.05)$} & \multicolumn{3}{|c|}{$\mathrm{T}=4.68 ; \mathrm{Y}=2.96$} & \multicolumn{3}{|c|}{$\mathrm{T}=1.23 ; \mathrm{Y}=0.78$} \\
\hline & \multicolumn{3}{|c|}{ Bacteria $\left(\mathrm{cfu} \mathrm{g}^{-1} \times 10^{5}\right){ }^{(7)} \mathrm{AH}$} & \multicolumn{3}{|c|}{ Fungi $\left(\mathrm{cfu} \mathrm{g}^{-1} \times 10^{4}\right) \mathrm{AH}$} \\
\hline Control & 20 & 21 & $21 \mathrm{C}$ & 5 & 6 & $6 \mathrm{D}$ \\
\hline SFWE at $10 \mathrm{~L} \mathrm{ha}^{-1}$ & 21 & 21 & $21 \mathrm{C}$ & 6 & 6 & $6 \mathrm{CD}$ \\
\hline SFWE at $20 \mathrm{~L} \mathrm{ha}^{-1}$ & 22 & 22 & $22 \mathrm{C}$ & 6 & 8 & $7 \mathrm{C}$ \\
\hline SFR at 4 ton $\mathrm{ha}^{-1}$ & 32 & 37 & $35 \mathrm{~B}$ & 9 & 15 & $12 \mathrm{~B}$ \\
\hline SFR at 6 ton $\mathrm{ha}^{-1}$ & 37 & 41 & $39 \mathrm{~A}$ & 12 & 18 & $15 \mathrm{~A}$ \\
\hline Mean (Y) & $26 \mathrm{~B}$ & $28 \mathrm{~A}$ & & $8 \mathrm{~B}$ & $11 \mathrm{~A}$ & \\
\hline \multirow[t]{2}{*}{$\operatorname{LSD}(\mathrm{p} \leq 0.05)$} & \multicolumn{3}{|c|}{$\mathrm{T}=2.04 ; \mathrm{Y}=1.29$} & \multicolumn{3}{|c|}{$\mathrm{T}=1.22 ; \mathrm{Y}=0.77$} \\
\hline & \multicolumn{3}{|c|}{ Alkaline phosphatase $\left(\mu \mathrm{g} \mathrm{NP} \mathrm{g}{ }^{-1}\right.$ soil $\left.^{-1}\right)$} & \multicolumn{3}{|c|}{ Dehydrogenase $\left(\mu \mathrm{g} \mathrm{TPFg}^{-1}\right.$ soil h$\left.^{-1}\right)$} \\
\hline Control & $135.14 \mathrm{e}$ & $135.16 \mathrm{e}$ & $135.15 \mathrm{C}$ & $22.35 \mathrm{~d}$ & $23.00 \mathrm{~d}$ & $22.67 \mathrm{C}$ \\
\hline SFWE at $10 \mathrm{~L} \mathrm{ha}^{-1}$ & $135.15 \mathrm{e}$ & $135.22 \mathrm{e}$ & $135.18 \mathrm{C}$ & $22.76 \mathrm{~d}$ & $23.26 \mathrm{~d}$ & $23.01 \mathrm{C}$ \\
\hline SFWE at $20 \mathrm{~L} \mathrm{ha}^{-1}$ & $135.45 \mathrm{e}$ & $135.52 \mathrm{e}$ & $135.48 \mathrm{C}$ & $23.00 \mathrm{~d}$ & $23.85 \mathrm{~d}$ & $23.43 \mathrm{C}$ \\
\hline SFR at 4 ton $\mathrm{ha}^{-1}$ & $162.25 \mathrm{~d}$ & $168.45 \mathrm{c}$ & $165.35 \mathrm{~B}$ & $29.00 \mathrm{c}$ & $35.00 \mathrm{~b}$ & $32.00 \mathrm{~B}$ \\
\hline SFR at 6 ton $\mathrm{ha}^{-1}$ & $180.25 \mathrm{~b}$ & $191.23 \mathrm{a}$ & $185.74 \mathrm{~A}$ & $34.00 \mathrm{~b}$ & $41.00 \mathrm{a}$ & $37.50 \mathrm{~A}$ \\
\hline Mean (Y) & $149.65 \mathrm{~B}$ & $153.12 \mathrm{~A}$ & & $26.22 \mathrm{~B}$ & $29.22 \mathrm{~A}$ & \\
\hline $\operatorname{LSD}(\mathrm{p} \leq 0.05)$ & \multicolumn{3}{|c|}{$\mathrm{T}=4.20 ; \mathrm{Y}=11.05 ; \mathrm{T} \times \mathrm{Y}=5.93$} & \multicolumn{3}{|c|}{$\mathrm{T}=2.18 ; \mathrm{Y}=1.38 ; \mathrm{T} \times \mathrm{Y}=3.09$} \\
\hline
\end{tabular}

Figures of interaction and main effects sharing the same case letter, do not differ significantly $(\mathrm{p} \leq 0.05)$ by the least significant difference test; likewise, the figures of main effects and interaction without lettering, do not differ significantly $(\mathrm{p} \leq 0.05)$ by the least significant difference test; ${ }^{(1)}$ Control= plots with no crop residues or extract application; (2) $\mathrm{SFWE}=$ sunflower water extract; (3) $\mathrm{SFR}=$ sunflower residues; (4) $\mathrm{T}=$ treatments; ${ }^{(5)} \mathrm{Y}=$ year; ${ }^{(6)} \mathrm{DAS}=$ days after sowing; ${ }^{(7)} \mathrm{AH}=$ after harvesting. 


\section{Weed dynamics}

Horse purslane (Trianthema portulacastrum) and purple nutsedge (Cyperus rotundus) were both dominant in each experimental unit in both years of study. This study indicated that density and dry weight of horse purslane differed significantly with various allelopathic weed management strategies. Total weed density and dry weight were also significantly different with various allelopathic weed management strategies. However, dry weight of purple nutsedge was nonsignificant among various allelopathic weed management strategies (Table 4). The year effect was significant for all weed parameters except for dry weight of purple nutsedge (Table 4). The interaction between allelopathic weed management strategies and year was significant for total weed density and density of (horse purslane and purple nutsedge) but non-significant for total dry weight and dry weight of purple nutsedge (Table 4).

Table 4 - Influence of sunflower water extract and residues on total weed density $\left(0.25 \mathrm{~m}^{-2}\right)$ and total weed dry weight $\left(\mathrm{g} 0.25 \mathrm{~m}^{-2}\right)$ in mung bean

\begin{tabular}{|c|c|c|c|c|c|c|}
\hline \multirow{2}{*}{ Treatment } & 2014 & 2015 & $\operatorname{Mean}(\mathrm{T})^{(4)}$ & 2014 & 2015 & Mean $(\mathrm{T})$ \\
\hline & \multicolumn{3}{|c|}{ Horse purslane density $\left(0.25 \mathrm{~m}^{-2}\right)$} & \multicolumn{3}{|c|}{ Horse purslane dry weight $\left(\mathrm{g} 0.25 \mathrm{~m}^{-2}\right)$} \\
\hline Control $^{(1)}$ & $42 \mathrm{a}$ & $42 \mathrm{a}$ & $42 \mathrm{~A}$ & 48 & 50 & $49 \mathrm{~A}$ \\
\hline SFWE at $10 \mathrm{~L} \mathrm{ha}^{-1(2)}$ & $38 \mathrm{a}-\mathrm{c}$ & $37 \mathrm{bc}$ & $38 \mathrm{~B}$ & 43 & 43 & $43 \mathrm{~B}$ \\
\hline SFWE at $20 \mathrm{~L} \mathrm{ha}^{-1}$ & $35 \mathrm{c}$ & $33 \mathrm{c}$ & $34 \mathrm{C}$ & 37 & 37 & $36 \mathrm{C}$ \\
\hline SFR at 4 ton $\mathrm{ha}^{-1(3)}$ & $26 \mathrm{~d}$ & $16 \mathrm{e}$ & $21 \mathrm{D}$ & 29 & 21 & $25 \mathrm{D}$ \\
\hline SFR at 6 ton ha ${ }^{-1}$ & $17 \mathrm{e}$ & $13 \mathrm{e}$ & $15 \mathrm{E}$ & 19 & 14 & $16 \mathrm{E}$ \\
\hline Mean $(Y)^{(5)}$ & $32 \mathrm{~A}$ & $28 \mathrm{~B}$ & & $35 \mathrm{~A}$ & $33 \mathrm{~B}$ & \\
\hline \multirow[t]{2}{*}{$\operatorname{LSD}(\mathrm{p} \leq 0.05)$} & \multicolumn{3}{|c|}{$\mathrm{T}=3.27 ; \mathrm{Y}=2.07 ; \mathrm{T} \times \mathrm{Y}=4.62$} & \multicolumn{3}{|c|}{$\mathrm{T}=0.92 ; \mathrm{Y}=1.33$} \\
\hline & \multicolumn{3}{|c|}{ Purple nutsedge density $\left(0.25 \mathrm{~m}^{-2}\right)$} & \multicolumn{3}{|c|}{ Purple nutsedge dry weight $\left(\mathrm{g} 0.25 \mathrm{~m}^{-2}\right)$} \\
\hline Control & $10 \mathrm{ab}$ & $10 \mathrm{a}$ & $10 \mathrm{~A}$ & 3 & 3 & 2 \\
\hline SFWE at $10 \mathrm{~L} \mathrm{ha}^{-1}$ & $9 \mathrm{ab}$ & $9 \mathrm{~b}$ & $9 \mathrm{~A}$ & 2 & 2 & 2 \\
\hline SFWE at $20 \mathrm{~L} \mathrm{ha}^{-1}$ & $8 \mathrm{c}$ & $7 \mathrm{~d}$ & $8 \mathrm{~B}$ & 2 & 2 & 2 \\
\hline SFR at 4 ton $\mathrm{ha}^{-1}$ & $6 \mathrm{e}$ & $6 \mathrm{f}$ & $6 \mathrm{C}$ & 1 & 1 & 2 \\
\hline SFR at 6 ton ha ${ }^{-1}$ & $5 \mathrm{f}$ & $3 \mathrm{~g}$ & $4 \mathrm{D}$ & 1 & 1 & 2 \\
\hline Mean (Y) & $8 \mathrm{~A}$ & $7 \mathrm{~B}$ & & 2 & 2 & \\
\hline \multirow[t]{2}{*}{$\operatorname{LSD}(\mathrm{p} \leq 0.05)$} & \multicolumn{3}{|c|}{$\mathrm{T}=0.59 ; \mathrm{Y}=0.37 ; \mathrm{T} \times \mathrm{Y}=5.07$} & \multicolumn{3}{|c|}{ NS } \\
\hline & \multicolumn{3}{|c|}{ Total weed density $\left(0.25 \mathrm{~m}^{2}\right)$} & \multicolumn{3}{|c|}{ Total weed dry weight $\left(\mathrm{g} 0.25 \mathrm{~m}^{-2}\right)$} \\
\hline Control & $57.29 \mathrm{a}$ & $57.89 \mathrm{a}$ & $57.59 \mathrm{~A}$ & 57.05 & 55.88 & $56.48 \mathrm{~A}$ \\
\hline SFWE at $10 \mathrm{~L} \mathrm{ha}^{-1}$ & $52.78 \mathrm{~b}$ & $52.27 \mathrm{~b}$ & $52.53 \mathrm{~B}$ & 53.79 & 49.39 & $51.59 \mathrm{~B}$ \\
\hline SFWE at $20 \mathrm{~L} \mathrm{ha}^{-1}$ & $44.10 \mathrm{c}$ & $43.88 \mathrm{c}$ & $43.99 \mathrm{C}$ & 45.77 & 38.02 & $41.89 \mathrm{C}$ \\
\hline SFR at 4 ton $\mathrm{ha}^{-1}$ & $37.32 \mathrm{~d}$ & $27.09 \mathrm{e}$ & $32.20 \mathrm{D}$ & 33.97 & 26.15 & $30.06 \mathrm{D}$ \\
\hline SFR at 6 ton ha ${ }^{-1}$ & $27.35 \mathrm{e}$ & $21.76 \mathrm{f}$ & $24.56 \mathrm{E}$ & 24.72 & 19.16 & $21.94 \mathrm{E}$ \\
\hline Mean (Y) & $43.77 \mathrm{~A}$ & $40.58 \mathrm{~B}$ & & $42.83 \mathrm{~A}$ & $37.95 \mathrm{~B}$ & \\
\hline $\operatorname{LSD}(\mathrm{p} \leq 0.05)$ & \multicolumn{3}{|c|}{$\mathrm{T}=3.58 ; \mathrm{Y}=2.27 ; \mathrm{T} \times \mathrm{Y}=1.63$} & \multicolumn{3}{|c|}{$\mathrm{T}=4.87 ; \mathrm{Y}=3.08$} \\
\hline
\end{tabular}

Figures of interaction and main effects sharing the same case letter, do not differ significantly $(\mathrm{p} \leq 0.05)$ by the least significant difference test; likewise, the figures of main effects and interaction without lettering, do not differ significantly ( $\mathrm{p} \leq 0.05)$ by the least significant difference test; ${ }^{(1)}$ Control= plots with no crop residues or extract application; ${ }^{(2)} \mathrm{SFWE}=$ sunflower water extract; ${ }^{(3)} \mathrm{SFR}=$ sunflower residues; ${ }^{(4)} \mathrm{T}=$ treatments; ${ }^{(5)} \mathrm{Y}=$ year.

The lowest densities for horse purslane $\left(13\right.$ plants $\left.0.25 \mathrm{~m}^{-2}\right)$ and purple nutsedge ( 3 plants $\left.0.25 \mathrm{~m}^{-2}\right)$ were recorded with sunflower residues at 6 ton ha ${ }^{-1}$ in the $2^{\text {nd }}$ year as compared to the control (42 and 10 plants $0.25 \mathrm{~m}^{-2}$, respectively). The lowest values were found in the control, and they were statistically similar to those of the sunflower water extracts at $10 \mathrm{~L} \mathrm{ha}^{-1}$ in the $1^{\text {st }}$ year (Table 4). Weed density and dry weight were decreased over time and the minimum values were

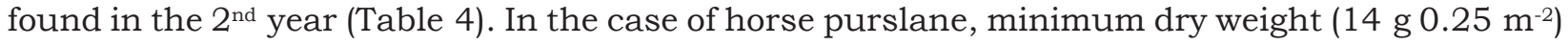
was found with sunflower residues at 6 ton ha $\mathrm{h}^{-1}$ followed by sunflower residues at 4 ton ha ${ }^{-1}$

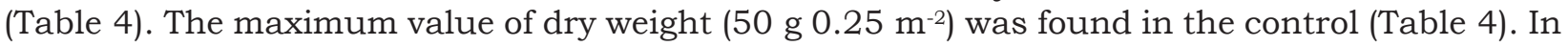


the case of total weed density, the interactive effect of allelopathic weed management strategies and year showed a statistically significant effect. The lowest total weed density (21.76) was recorded with sunflower residues at 6 ton ha ${ }^{-1}$ in the $2^{\text {nd }}$ year as compared to the control

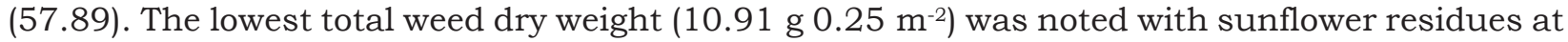

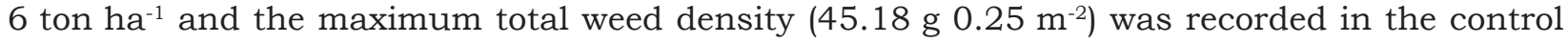
(Table 4).

\section{Yield components}

Yield and yield components (number of pods per plant, number of seeds per pod, 1000 seed weight) differed significantly among the various allelopathic weed management strategies (Table 5). Likewise, the year effect was significant for all yield and yield components (Table 5). The interaction between allelopathic weed management strategies and year was significant for number of pods per plant and yield (Table 5). However, the interaction was non-significant for no. of seed per pod and weight of 1000 seeds (Table 5).

The results indicated that the maximum (26.13) numbers of pods per plant were noted with sunflower residues at 6 ton $\mathrm{ha}^{-1}$ in the $2^{\text {nd }}$ year as compared to the control (15.33). Among allelopathic weed management strategies, the maximum value of number of seeds per pod (9.60), 1000 -seed weight $(54.99 \mathrm{~g})$ and yield $\left(1014.3 \mathrm{~kg} \mathrm{ha}^{-1}\right)$ were recorded with sunflower residues at 6 ton ha-1. The minimum value of number of seeds per pod (5.93), 1000 seed weight (50.40 g) and yield $\left(746.0 \mathrm{~kg} \mathrm{ha}^{-1}\right)$ were found in the control (Table 5). There was a linear increase in number of pods per plant, number of seeds per pod, 1000-seed weight and yield over time and all the above-mentioned values were significantly increased in the $2^{\text {nd }}$ year of study (Table 5). Among all treatments sunflower residue at 6 tons ha-1 gave maximum economical returns (339\$) during both years. While, minimum net benefit was obtained from control (Table 6).

Incorporation of allelopathic residues is a green approach for improving soil health and weed management in field crops. The results indicated that increased quantities of crop residues decreased bulk density and increased total porosity of soil over time (Table 2). Shaver (2010) reported that soil porosity is directly related to soil bulk density because as soil bulk density decreases, soil porosity increases. In the case of soil properties, sunflower residues as allelopathic

Table 5 - Influence of sunflower water extract and residues on yield and yield components of mung bean

\begin{tabular}{|c|c|c|c|c|c|c|}
\hline \multirow{2}{*}{ Treatment } & 2014 & 2015 & $\operatorname{Mean}(\mathrm{T}){ }^{(4)}$ & 2014 & 2015 & Mean $(\mathrm{T})$ \\
\hline & \multicolumn{3}{|c|}{ No. of pods per plant } & \multicolumn{3}{|c|}{ No. of seeds per pod } \\
\hline Control $^{(1)}$ & $14.13 \mathrm{f}$ & $15.33 \mathrm{ef}$ & $14.73 \mathrm{E}$ & 5.48 & 6.37 & $5.93 \mathrm{C}$ \\
\hline SFWE at $10 \mathrm{~L} \mathrm{ha}^{-1(2)}$ & $17.01 \mathrm{de}$ & $18.38 \mathrm{~d}$ & $17.70 \mathrm{D}$ & 6.55 & 7.13 & $6.84 \mathrm{~B}$ \\
\hline SFWE at $20 \mathrm{~L} \mathrm{ha}^{-1}$ & $18.45 \mathrm{~d}$ & $21.25 \mathrm{bc}$ & $19.85 \mathrm{C}$ & 6.95 & 7.35 & $7.15 \mathrm{~B}$ \\
\hline SFR at 4 ton $\mathrm{ha}^{-1(3)}$ & $19.03 \mathrm{~cd}$ & $24.11 \mathrm{a}$ & $21.57 \mathrm{~B}$ & 7.07 & 7.68 & $7.38 \mathrm{~B}$ \\
\hline SFR at 6 ton $\mathrm{ha}^{-1}$ & $21.55 \mathrm{~b}$ & $26.13 \mathrm{a}$ & $23.84 \mathrm{~A}$ & 9.24 & 9.96 & $9.60 \mathrm{~A}$ \\
\hline Mean $(\mathrm{Y})^{(5)}$ & $18.03 \mathrm{~B}$ & $21.04 \mathrm{~A}$ & & $7.06 \mathrm{~B}$ & $7.70 \mathrm{~A}$ & \\
\hline \multirow[t]{2}{*}{$\operatorname{LSD}(\mathrm{p} \leq 0.05)$} & \multicolumn{3}{|c|}{$\mathrm{T}=1.63 ; \mathrm{Y}=1.03 ; \mathrm{T} \times \mathrm{Y}=2.30$} & \multicolumn{3}{|c|}{$\mathrm{T}=0.81 ; \mathrm{Y}=0.51$} \\
\hline & \multicolumn{3}{|c|}{ 1000-seed weight $(\mathrm{g})$} & \multicolumn{3}{|c|}{ Yield $\left(\mathrm{kg} \mathrm{ha}^{-1}\right)$} \\
\hline Control & 50.26 & 50.54 & $50.40 \mathrm{D}$ & $745.3 \mathrm{e}$ & $746.7 \mathrm{e}$ & $746.0 \mathrm{E}$ \\
\hline SFWE at $10 \mathrm{~L} \mathrm{ha}^{-1}$ & 52.58 & 53.35 & $52.97 \mathrm{C}$ & $789.2 \mathrm{~d}$ & $801.5 \mathrm{~d}$ & $795.4 \mathrm{D}$ \\
\hline SFWE at $20 \mathrm{~L} \mathrm{ha}^{-1}$ & 53.25 & 53.67 & $53.46 \mathrm{~B}$ & $844.1 \mathrm{c}$ & $854.4 \mathrm{c}$ & $849.3 \mathrm{C}$ \\
\hline SFR at 4 ton $\mathrm{ha}^{-1}$ & 53.76 & 53.76 & $53.76 \mathrm{~B}$ & $934.2 \mathrm{~b}$ & $925.4 \mathrm{~b}$ & $929.8 \mathrm{~B}$ \\
\hline SFR at 6 ton $\mathrm{ha}^{-1}$ & 54.49 & 55.48 & $54.99 \mathrm{~A}$ & $1009.1 \mathrm{a}$ & $1019.4 \mathrm{a}$ & $1014.3 \mathrm{~A}$ \\
\hline Mean (Y) & $52.87 \mathrm{~B}$ & $53.36 \mathrm{~A}$ & & $864.38 \mathrm{~B}$ & $869.49 \mathrm{~A}$ & \\
\hline $\operatorname{LSD}(\mathrm{p} \leq 0.05)$ & \multicolumn{3}{|c|}{$\mathrm{T}=0.35 ; \mathrm{Y}=0.37$} & \multicolumn{3}{|c|}{$\mathrm{T}=14.75 ; \mathrm{Y}=4.33 ; \mathrm{T} \times \mathrm{Y}=20.85$} \\
\hline
\end{tabular}

Figures of interaction and main effects sharing the same case letter, do not differ significantly $(\mathrm{p} \leq 0.05)$ by the least significant difference test; likewise, the figures of main effects and interaction without lettering, do not differ significantly $(\mathrm{p} \leq 0.05)$ by the least significant difference test; ${ }^{(1)}$ Control= plots with no crop residues or extract application; ${ }^{(2)} \mathrm{SFWE}=$ sunflower water extract; ${ }^{(3)} \mathrm{SFR}=$ sunflower residues; ${ }^{(4)} \mathrm{T}=$ treatments; ${ }^{(5)} \mathrm{Y}=$ year. 
ULLAH, R. et al. Sunflower residue incorporation suppresses weeds, enhances soil properties and seed yield of ...

Table 6 - Economics of mung bean grown in various allelopathic weed management strategies

\begin{tabular}{|c|c|c|c|c|c|c|}
\hline Treatment & $\begin{array}{c}\text { Yield } \\
\left(\mathrm{kg} \mathrm{ha}^{-1}\right)\end{array}$ & $\begin{array}{l}\text { Adjusted } \\
\text { yield } \\
\left(\mathrm{kg} \mathrm{ha}^{-1}\right)\end{array}$ & $\begin{array}{l}\text { Gross income } \\
\qquad \mathrm{ha}^{-1(5)}\end{array}$ & $\begin{array}{c}\text { Total cost } \\
\$ \mathrm{ha}^{-1}\end{array}$ & $\begin{array}{c}\text { Net benefits } \\
\qquad \mathrm{ha}^{-1}\end{array}$ & $\begin{array}{c}\text { Benefit cost } \\
\text { ratio }\end{array}$ \\
\hline Control $^{(1)}$ & 746 & 671 & 752 & 615 & 137 & 0.22 \\
\hline SFWE at $10 \mathrm{~L} \mathrm{ha}^{-1(2)}$ & 795 & 716 & 802 & 625 & 177 & 0.28 \\
\hline SFWE at $20 \mathrm{~L} \mathrm{ha}^{-1}$ & 849 & 764 & 856 & 628 & 229 & 0.36 \\
\hline SFR at 4 ton $\mathrm{ha}^{-1}(3)$ & 930 & 837 & 937 & 663 & 274 & 0.41 \\
\hline SFR at 6 ton $\mathrm{ha}^{-1}$ & 1014 & 913 & 1022 & 683 & 339 & 0.50 \\
\hline Remarks & $\$ 44.67 / 40 \mathrm{~kg}$ & $\begin{array}{c}10 \% \text { less than } \\
\text { actual }\end{array}$ & $\$$ ha $^{-1}$ & $\$ \mathrm{ha}^{-1}$ & $\$ \mathrm{ha}^{-1}$ & $\begin{array}{l}1 \$=98.5 \\
\operatorname{PKR}^{(4)}\end{array}$ \\
\hline
\end{tabular}

(1) Control= plots with no crop residues or extract application; (2) SFWE= sunflower water extract; (3) $\mathrm{SFR}=$ sunflower residues; (4) $\mathrm{PKR}=$ Pakistani rupees; ${ }^{(5)} \$=$ US dollar.

weed management strategy improved SOM, N, available K and P in the soil (Table 2). Crop residues are good sources of nutrients and are the primary source of organic material added to soil. They increase nutrient availability and water holding capacity of soils (Krishna et al., 2004). Moisture retention is the main benefit of residue incorporation. It is caused by a decrease in runoff and evaporation of water from the soil (Verhulst et al., 2011). The improvement in nutrient accumulation (especially $\mathrm{P}$ and $\mathrm{K}$ ) might be attributed to the enhanced moisture retention within the soil due to residue incorporation that helped to increase the solubility of nutrients in the soil Residue incorporation improved nutrient accumulation (especially P and K) and enhanced the moisture holding capasity of soil (Zhou et al., 2002). Improved moisture availability resulting from residue incorporation also indicated that water holding capacity of the soil was improved and soil moisture was available for a longer time for support to plant growth (Jin et al., 2013). This increase in moisture retention properties might decrease the irrigational requirement of the crops, which should be investigated in future studies. In a previous study, Raut et al. (2010) stated that incorporation of sunflower straw at 4 ton ha ${ }^{-1}+\mathrm{RDF}$ at $(125 \% \mathrm{~N}+100 \% \mathrm{P})$ in green gram recorded significantly higher values for soil $\mathrm{N}, \mathrm{K}$, and $\mathrm{P}$ content in a green gram-sunflower sequence.

In our study, there was a linear decrease in soil $\mathrm{pH}$ after application of sunflower residues as an allelopathic weed management strategy (Table 2). Gong et al. (2008) reported that sunflower oil residue incorporation into the soil decreases soil $\mathrm{pH}$. Residues of Parthenium hysterophorus in the soil also changed soil chemistry. It was found that the $\mathrm{pH}$ of $P$. hysterophorus infested soil decreased, whereas the EC of soil increased (Batish et al., 2002a, b).

Using sunflower residues as an allelopathic weed management strategy in mung bean improved the microbial population and enzymatic activities of the soil (Table 3). Microbial abundance and soil enzymes are soil biological activities which are one of the most important indicators of soil quality (Dick, 1994). The incorporation of residues of different crops in the soil modified its bio-chemical attributes i.e. soil microbial population and soil enzymatic activity (Doran, 1980; Dick et al., 1983). Soil enzymes and micro biota play a key role in nutrient availability. Dehydrogenase enzyme is important for oxidation of soil organic matter (SOM). It transfers hydrogen and electrons from substrates to acceptors. The activity of soil enzymes viz. dehydrogenase and phosphatase depends on type of residues incorporated into the soil. It also depends on the moisture contents and temperature of the soil. It affects the activity of dehydrogenase by changing the oxidation reduction status of the soil (Brzezinska et al., 1998). Incorporation of crop residues viz. tobacco and sunflower into the soil increased the activities of most enzymes in the soil, while the tomato crop residues only increased the activity of amylase and phosphodiesterase. In Akola, Maharashtra, Ravankar et al. (2000) reported that incubation of soil with $1 \%$ organic residues of each cotton stalk, safflower straw, sorghum stubble, soybean stover, wheat straw, sugar cane trash, ground nut husk, sunflower straw, green gram stover, parthenium, and xanthium residues with seeds showed wide variation in the rate of decomposition, $\mathrm{C}: \mathrm{N}$ ratio and microbial population at different intervals. Populations of fungi, 
bacteria, and actinomycetes increased at 30 days of incubation. Bacteria were predominant over fungi and actinomycetes.

The results showed that sunflower residue incorporation improved soil health and suppressed weeds. This sustainable approach caused a significant reduction in weed density and dry weight of weed species in mung bean (Table 4) owing to presence of phenolic compounds (cholorogenic, caffiec, syringic, vanilic and ferulic acids) and terpinoids (sesquiterpene lactones) with a wide spectrum of biological activities including allelopathy (Anjum and Bajwa, 2005; Marsni et al., 2015). In the case of field crops, sunflower had the highest allelopathic potential (Alsaadawi et al., 2007). The treatments with sunflower residues showed the highest suppression of weeds as compared to treatments with sunflower water extracts (Table 4). Kandhro et al. (2015) and Farooq et al. (2011) reported a significant reduction in weed density $(25.26 \%)$ and dry weight $(14.60 \%)$ resulting from the application of sunflower water extract. The highest suppression of weeds was recorded in the wheat field as a result of sunflower residue incorporation because of the release of allelochemicals as well as the reduction of light penetration (Reberg-Horton et al., 2005; Alsaadawi et al., 2007). Crop residues may change the frequency and distribution of weeds, and they suppress weed emergence and weed growth (Essien et al., 2009; Khaliq et al., 2015). Previously, Zaji and Majd (2011) showed that the fresh and dry weights of different weeds biota viz. red root pig weed (Amaranthus retroflexu), palmer amaranth (Amaranthus palmeri), black nightshade or wonder berry (Solanum nigrum) and curled dock (Rumex crispus) were severely decreased by the impact of canola crop residues. Growth suppression of dominant weeds biota viz. purple nutsedge and horse purslane in this study might have been due to physical resistance through incorporation of sunflower residues or release of chemicals from these residues. The release of allelochemicals through different parts of plants are dependent on many factors i.e. crop family, size and dose of mulching, decomposition rate, moisture contents, texture of soil and soil micro biota (Kamara et al., 2000; Khaliq et al., 2014).

The present study proved that there was more than 36\% increase in mung bean yield through effective allelopathic weed management strategies. These increases in crop yield might have been due to the improvement of soil health and weed suppression during the critical growth period of the crop. The effective suppression of weeds also increases the availability of resources such as light, water, nutrients and space (Kruidhof et al., 2008). In a recent study on the application of wheat residues in the Mediterranean environment by Stagnari et al. (2014), it was found that soil moisture conservation ability was improved especially during the critical growth period of the test crop. The residues which are completely decomposed in the soil not only provide allelochemicals but also participate in crop nutrition. They provide nitrogen through release into the rhizosphere of the tested crop plant. Through the application of residues as a biological weed management strategy, it immobilizes nitrogen, which may decrease the immediate supply of nitrogen (Khaliq et al., 2015). However, at later stages of crop growth, the availability of nitrogen was enhanced through mineralization, proving that the prolonged supply of nitrogen is a continuous source of nutrition for the test crop as well as the next crops.

Thus, sunflower residue incorporation improves soil moisture retention, enhances nutrient cycling and microbial activity (Alam et al., 2014; Adugna and Abegaz, 2016; Nawaz et al., 2016), and suppresses weeds by physical hindrance, reducing light penetration, and through release of allelochemicals from such residues (Kamara et al., 2000; Khaliq et al., 2014). Because of the above-mentioned advantages of sunflower residues, better seed yield and higher profitability were achieved in spring-planted mung bean in 2014-15.

Allelopathic crop residues are a valuable source of nutrition and play an efficient role in controlling weeds, which is essential for sustainable crop production. In our study, there was differential ability to suppress weeds among various treatments application of sunflower residues and water extracts. There was high suppression of weed density and dry weight when sunflower residues at 6 ton ha ${ }^{-1}$ were incorporated into the soil. Apparently, the residues favorably affected the soil properties viz. nutrient dynamics, microbial populations, soil enzyme activities. The improvement in soil properties and suppression in weeds resulted in better seed yield and achieved higher profitability in spring-planted mung bean. In short, sunflower residue incorporation may provide multidimentional benefits for better weed control, enhanced soil health and higher seed yield of spring-planted mung bean. 


\section{ACKNOWLEDGEMENTS}

The financial support from Higher Education Commission Pakistan (HEC) under project no. 20-2014/NRPU/R\&D/12/4188 is highly acknowledged.

\section{REFERENCES}

Adugna A., Abegaz A. Effects of land use changes on the dynamics of selected soil properties in northeast Wellega, Ethiopia. Soil. 2016;2:63-70.

Aktar S. et al. Efficacy of herbicides in controlling weeds in mung bean (Vigna radiata L. Wilczek) field. Agriculturists. 2015;13:127-32.

Alam M.K. et al. Effect of tillage practices on soil properties and crop productivity in wheat-mungbean-rice cropping system under subtropical climatic conditions. Sci World J. 2014;1:1-15.

Alsaadawi I.S. et al. Differential allelopathic potential of grain sorghum genotypes to weed. Allelop J. 2007;19:153-60.

Anjum T., Bajwa R. A bioactive annuionone from sunflower leaves. Phytochemistry. 2005;66:1919-21.

Ashiq M., Cheema Z.A. Effective use of herbicides. Faisalabad: Weed Science Allelopathy Laboratory, University of Agriculture Faisalabad, 2005. 7p.

Aslam Z. et al. Lysobacter oryzae sp. nov., isolated from the rhizosphere of rice (Oryza sativa L.) managed under no-tillage practice. Int J Syst Evol Microbiol. 2008;59:675-80.

Bai Z.G. et al. Global assessment of land degradation and improvement; identification by remote sensing; Report 2008/01. Wageningen: ISRIC-World Soil Information, 2008.

Bajgai Y. et al. Comparison of organic and conventional managements on yields, nutrients and weeds in a corn-cabbage rotation. Renewable Agric Food Syst. 2015;30:132-42.

Batish D.R. et al. Phytotoxic effect of parthenium residues on the selected soil properties and growth of chickpea and radish. Weed Biol Manage. 2002a;2:73-8.

Batish D.R. et al. Phytotoxicity of sunflower residues against some summer season crops. J Agron Crop Sci. 2002b;188:19-24.

Blake G.R., Hartge K.H. Bulk density. In: Methods of soil analysis. In: Klute A. editor. Physical and mineroalogical methods. $2^{\text {nd }}$. ed. Madison: American Society of Agronomy, 1986. Part 1. p.363-75. (Agronomy Monograph, 9)

Bremner J.M., Mulvaney C.S. Total nitrogen. In: Page A.L. editors. Methods of soil analysis. Madison: American Society of Agronomy and Soil Science Society of America, 1982. Pt. 2. p.1119-23.

Brzezinska M. et al. Soil oxygen status and dehydrogenase activity. Soil Biol Biochem. 1998;30:1783-90.

Dick R.P. Soil enzymatic activities as in indicator of soil quality. In: Doran J.W. et al. editors. Definding soil quality for a sustainable development. Madison: Soil Science Society of America, 1994. p.107-24.

Dick W.A. et al. Effects of soils on acid phosphatase and inorganic pyro phosphatase of corn roots (Zea mays). Soil Sci. $1983 ; 136: 19-25$.

Doran J.W. Microbial changes associated with residue management with reduce management with reduced tillage. Soil Sci Soc Am J. 1980;44:518-24.

Essien B. et al. Effect of organic mulch materials on maize performance and weed growth in the derived savanna of South Eastern Nigeria. Nigeria Agric J. 2009;40:1-9.

Fahad S. et al. Weed growth and crop yield loss in wheat as influenced by row spacing and weed emergence times. Crop Prot. 2015;71:101-8.

Farooq M. et al. The role of allelopathy in agricultural pest management. Pest Manag Sci. 2011;67:493-506. 
Gong Z. et al. Effects of vegetable oil residue after soil extraction on physical-chemical properties of sandy soil and plant growth. J Environ Sci. 2008;20:1458-62.

Government of Pakistan. Economic Survey of Pakistan 2015-2016. Islamabad: Finance Division, Economic Advisory Wing, 2016. 30p.

Helmke P.A., Sparks D.L. Lithium, sodium, potassium, rubidium, and cesium. In: Sparks D.L. et al. Methods of soil analysis; Chemical Methods. Madison: SSSA Book, 1996. Pt 3. p.551-75.

Hozayn M. et al. Crop residues, an effective tool for improving growth of wheat and suppression of some associated weeds. Allelop J. 2011;27:237-344.

Hussain S. et al. Rice management interventions to mitigate greenhouse gas emissions: a review. Environ Sci Poll Res. 2015;22:3342-60.

Janssen P.H. et al. Improved culturability of soil bacteria and isolation in pure culture of novel members of the divisions Acido bacteria, Actino bacteria, Proteo bacteria, and Verruco microbia. Appl Environ Microbiol. 2002;68:2391-6.

Jin Y.Q. et al. Effects of maize straw returning on water dynamics and water use efficiency of winter wheat in lime concretion black soil. J Triticeae Crops. 2013;33:1-7.

Johnson J.M.P. et al. Agricultural opportunities to mitigate greenhouse gas emissions. Environ. Poll. 2007;150:107-24.

Kamara A. et al. Selective control of weeds in an arable crop by mulches from some multipurpose trees in south western Nigeria. Agrofor Syst. 2000;50:17-26.

Kandhro M.N. et al. Effect of allelopathic water extract of sorghum and sunflower on weed mortality and cotton yield. Sarhad J Agric. 2015;31:165-74.

Khaliq A. et al. Swine cress (Cronopus didymus L. Sm.) residues inhibit rice emergence and early seedling growth. Phillips Agric Sci. $2014 ; 96: 419-25$.

Khaliq A. et al. Wheat residue management options affect productivity, weed growth and soil properties in direct-seeded fine aromatic rice. Clean-Soil Air Water. 2015;43:1259-65.

Khaliq A. et al. Supplementing herbicides with manual weeding improves weed control efficiency, growth and yield of direct seeded rice. Int J Agric Biol. 2013;15:191-9.

Kong C.H. et al. Impact of allelochemical exuded from allelopathic rice on soil microbial community. Soil Biol Biochem. 2008;40:1862-9.

Krishna G.M.A. et al. Rice residue management options and effects on soil properties and crop productivity. Food Agric Environ. 2004;2:224-31.

Kruidhof H. et al. Ecological weed management by cover cropping: effects on weed growth in autumn and weed establishment in spring. Weed Res. 2008;48:492-502.

Malhi S., Lemke R. Tillage crop residue and N fertilizer effects on crop yield, nutrient uptake, soil quality and nitrous oxide gas emissions in a second 4-yr rotation cycle. Soil Till Res. 2007;96:269-83.

Marsni Z.E. et al. Isolation of bioactive compounds from sunflower leaves (Helianthus annuus L.) extracted with supercritical carbon dioxide. J Agric Food Chem. 2015;63:6410-21.

Martin J.P. Use of acid, rose bengal and streptomycin in the plate method for enumerating soil fungi. Soil Sci. 1950;69:215-32.

Min H. et al. Effects of butachlor on microbial populations and enzyme activities in paddy soil. J Environ Sci Health. 2001;36:581-95.

Nawaz A. et al. Mulching affects soil properties and greenhouse gases emissions under long term no-till and plough till systems in Alfisol of Central Ohio. Land Develop Degrad. 2016;28:673-81.

Olsen S.O., Sommers I.E. Phosphorus. In: Page A.L. editor. Methods of soil analysis. $2^{\text {nd }}$. ed. Chemical and microbial properties. Madison: American Society of Agronomy, 1982. Pt 2. p.403-30. 
Pandey J., Mishra B.N. Effect of weed management practices in a rice mustard-mungbean cropping system on weeds and yield of crops. Ann Agric Res. 2003;24:737-42.

Rab A. et al. Impact of bio char on mungbean yield and yield components. Pure Appl Biol. 2016;5:632-40.

Raut V.U. et al. Effect of crop residues on nutrient contents in green gram-sunflower cropping sequence. Green Farm. 2010;1:14-9.

Ravankar H.N. et al. Decomposition of different organic residues in soil. PKV Res J. 2000;24:23-5.

Reberg-Horton S.C. et al. Changes over time in the allelochemicals content of ten cultivars of rye (Secale cereale). J Chemic Ecol. 2005;31:179-93.

Ruiyu L. et al. Impact of allelopathic rice seedlings on rhizospheric microbial populations and their functional diversity. Acta Ecol Sinica. 2007;27:3644-54.

Ryan J. et al. Soil and plant analysis: laboratory manual. Aleppo: International Center for Agricultural Research in Dry Areas (ICARDA), 2001. 172p.

Sharma P. et al. Impact of tillage and mulch management on economics, energy requirement and crop performance in maize-wheat rotation in rainfed subhumid inceptisols. India Eur J Agron. 2011;34:46-51.

Shaver T. Crop residue and soil physical properties. In: Proceedings of the 22nd Annual Central Plains Irrigation Conference. Kearney, NE, Feb 24-25, 2010.

Stagnari F. et al. Effects of straw mulch on growth and yield of durum wheat during transition to conservation agriculture in Mediterranean environment. Field Crop Res. 2014;167:51-63.

Steel R.G.D. et al. Principles and procedures of statistics: A biometric approach. $3^{\text {rd }}$.ed. New York: McGraw Hill Book, 1996.

Tabatabai M.A., Bremner J.M. Use of p-nitrophenyl phosphate for assay of soil phosphatase activity. Soil Biol Biochem. 1969;1:301-7.

Tariq I.M. et al. Pesticides exposure in Pakistan: a review. Environ Inter. 2007;33:1107-22.

US Salinity Laboratory Staff. Diagnosis and improvement of saline and alkali soils. USDA Agricultural Handbook No. 60. Washington, DC.: US Government Printing Office. 1954.

Verhulst N. et al. Soil water content, maize yield and its stability as affected by tillage and crop residue management in rainfed semi arid highlands. Plant Soil. 2011;124:347-56.

Vomocil J.A. Porosity. In: Blake C.A. editor. Methods of soil analysis. Madison: American Society of Agronomy, 1965. p.299-314.

Walkley A., Black I.A. An examination of Degtjareff method for determining soil organic matter and a proposed modification of the chromic acid titration method. Soil Sci. 1934;37:29-38.

Weston L.A., Duke S.O. Weed and crop allelopathy. Crit Rev Plant Sci. 2003;22:367-89.

World Resources Institute - WRI. Creating a sustainable food future. Report 2013-2014: Interim Findings. Washington, DC: World Resources Institute, 2014.

Wu F. et al. Effect of cinnamic acid on soil microbial characteristics in the cucumber rhizosphere. Europ J Soil Biol. 2009;45:356-62.

Wu W.X. et al. Bt-transgenic rice straw affects the culturable micro biota and dehydrogenase and phosphatase activities in a flooded paddy soil. Soil Biol Biochem. 2004;36:289-95.

Zaji B., Majd A. Allelopathic potential of canola (Brassica napus L.) residues on weed suppression and yield response of maize (Zea mays L.) In: International Conference on Chemical, Ecology and Environmental Sciences (IICCEES). Pattaya: 2011. p.457-60.

Zhou J. et al. Study of comprehensive utilization efficiency of returning rice straw to field. Chinese Agric Sci Bull. 2002;4:003. 